\title{
Discrimination of Poly(vinyl chloride) Samples with Different Plasticizers and Prediction of Plasticizer Contents in Poly(vinyl chloride) Using Near-infrared Spectroscopy and Neural-network Analysis
}

\author{
Kazumitsu SAEKI, ${ }^{* \dagger}$ Kimito Funatsu, $* *$ and Kazutoshi TAnabE*** \\ *Toyama Industrial Technology Center, 150 Futagami-machi, Takaoka, Toyama 933-0981, Japan \\ **Department of Knowledge-based Information Engineering, Toyohashi University of Technology, \\ 1-1 Hibarigaoka, Tempaku, Toyohashi, Aichi 441-8580, Japan \\ ***Department of Management Information Science, Chiba Institute of Technology, \\ 2-17-1 Tsudanuma, Narashino, Chiba 275-0016, Japan
}

\begin{abstract}
In the recycling of poly(vinyl chloride) (PVC), it is required to discriminate every plasticizer for quality control. For this purpose, the near-infrared spectra were measured for 41 kinds of PVC samples with different plasticizers (DINP, DOP, DOA, TOTM and Polyester) and different plasticizer contents $(0-49 \%)$. A neural-network analysis was applied to the near-infrared spectra pretreated by second-derivative processing. They were discriminated from one another. The neuralnetwork analysis also allowed us to propose a calibration model which predicts the contents of plasticizers in PVC. The correlation coefficient (R) and the root-mean-square error of prediction (RMSEP) for the DINP calibration model were found to be 0.999 and $0.41 \mathrm{wt} \%$, respectively. In comparison, a partial least-squares regression analysis was carried out. The R and RMSEP of the DINP calibration model were calculated to be 0.993 and $1.27 \mathrm{wt} \%$, respectively. It is found that a near-infrared spectra measurement combined with a neural-network analysis is useful for plastic recycling.
\end{abstract}

(Received September 17, 2002; Accepted November 22, 2002)

\section{Introduction}

Poly(vinyl chloride) (PVC) is one of the most widely used materials among plastics. PVC is used for various products, such as pipes, wire coverings and agricultural sheets. Much PVC with a plasticizer has been manufactured for additives with different properties. A great number of plasticizers, such as dioctyl phthalate, diisononyl phthalate, are produced.

In plastic recycling, plastic wastes have to be sorted according to their types and, even more, the same kinds of plastics are to be discriminated according to their grades or additives. In the case of PVC recycling, it is absolutely essential that PVC wastes are sorted according to every plasticizer.

A plasticizer in PVC is generally measured by gas chromatography (GC), mass spectroscopy (MS) and nuclear magnetic resonance (NMR) analyses. Although speedy and intact analyses of plasticizers are indispensable to the recycling of plastics, plasticizers cannot be analyzed quickly and nondestructively by these methods.

Near-infrared (NIR) studies of polymers already stretch back more than two decades. ${ }^{1-13}$ Since multivariate data analysis and optical light fibers were introduced to the NIR region, the usefulness of NIR spectroscopy in polymer research and analysis has increased dramatically. NIR spectroscopy has advantages in terms of rapid and non-destructive measurements. Although NIR spectra measurements are applicable to plastic recycling, the amount of information in the NIR spectra is little. Neural networks have been applied to a wide range of problems in the field of chemistry due to their advantages over other methods: ${ }^{14,15}$ the capability of analyzing non-linear relationships between structures and spectra; the ability to achieve rapid processing due to their parallel algorithms; and flexibility in processing applicable to fluctuating data. For these reasons, papers have been published on the analysis of spectra with neural networks.

We have already succeeded to develop techniques to discriminate more than 50 different kinds of plastic patterns, ${ }^{16}$ and to separate polyethylene grades. ${ }^{17}$ In the present paper, we demonstrate methods which discriminate PVC with different plasticizers and predict the plasticizer content in PVC by combining NIR spectroscopy and neural-networks analysis.

\section{Experimental}

Samples

Forty-one kinds of PVC samples with various plasticizers and different plasticizer contents $(0-49$ wt $\%)$ used in this study were obtained from TOYOX CO., LTD. (Toyama, Japan). Dioctyl phthalate (DOP), diisononyl phthalate (DINP), tri-octyl trimellitate (TOTM), di-octyl adipate (DOA) and polyester type (Polyester) were used for the plasticizers. Sample components and compositions used in this study are listed in Table 1.

† To whom correspondence should be addressed. 
Table 1 Kinds of plasticizers and contents of plasticizers of PVC samples examined in the present study

No. Kind of plasticizer Content of plasticizer, Content of PVC,

\begin{tabular}{|c|c|c|c|}
\hline & & $\mathrm{wt} \%$ & wt $\%$ \\
\hline 1 & None & 0 & 100 \\
\hline 2 & DINP & 11 & 89 \\
\hline 3 & DINP & 19 & 81 \\
\hline 4 & DINP & 26 & 74 \\
\hline 5 & DINP & 32 & 68 \\
\hline 6 & DINP & 37 & 63 \\
\hline 7 & DINP & 42 & 58 \\
\hline 8 & DINP & 46 & 54 \\
\hline 9 & DINP & 49 & 51 \\
\hline 10 & DOP & 11 & 89 \\
\hline 11 & DOP & 19 & 81 \\
\hline 12 & DOP & 26 & 74 \\
\hline 13 & DOP & 32 & 68 \\
\hline 14 & DOP & 37 & 63 \\
\hline 15 & DOP & 42 & 58 \\
\hline 16 & DOP & 46 & 54 \\
\hline 17 & DOP & 49 & 51 \\
\hline 18 & DOA & 11 & 89 \\
\hline 19 & DOA & 19 & 81 \\
\hline 20 & DOA & 26 & 74 \\
\hline 21 & DOA & 32 & 68 \\
\hline 22 & DOA & 37 & 63 \\
\hline 23 & DOA & 42 & 58 \\
\hline 24 & DOA & 46 & 54 \\
\hline 25 & DOA & 49 & 51 \\
\hline 26 & TOTM & 11 & 89 \\
\hline 27 & TOTM & 19 & 81 \\
\hline 28 & TOTM & 26 & 74 \\
\hline 29 & TOTM & 32 & 68 \\
\hline 30 & TOTM & 37 & 63 \\
\hline 31 & TOTM & 42 & 58 \\
\hline 32 & TOTM & 46 & 54 \\
\hline 33 & TOTM & 49 & 51 \\
\hline 34 & Polyester & 11 & 89 \\
\hline 35 & Polyester & 19 & 81 \\
\hline 36 & Polyester & 26 & 74 \\
\hline 37 & Polyester & 32 & 68 \\
\hline 38 & Polyester & 37 & 63 \\
\hline 39 & Polyester & 42 & 58 \\
\hline 40 & Polyester & 46 & 54 \\
\hline 41 & Polyester & 49 & 51 \\
\hline
\end{tabular}

\section{Instrument}

The NIR spectra in the region of $1.2-2.4 \mu \mathrm{m}$ were measured for a sheet with PlaScan-SH (Opt Research Inc., Tokyo, Japan). The number of data points for each NIR spectrum of the above region was 1200. A scan for an NIR spectrum using this device can be carried out within less than $1 \mathrm{~s}$.

\section{Data analysis}

NIR spectra were measured three times for each sample with different sample orientations. The total number of recorded spectra was 123. The NIR spectra in the region of $1.2-2.4 \mu \mathrm{m}$ were subjected to the following pretreatments before training a neural network: (1) the maximum and minimum absorbances in each spectrum were normalized to 1.0 and 0.0 , respectively, (2) the data of 1200 points were averaged at every 10 points (reduced to 120 points in total), (3) second-derivative spectra were calculated, and (4) the maximum absolute value was normalized to 1.0 again. Figures 1(a)-(d) illustrate the averaged and normalized spectra of PVC with 2 different plasticizers, DOP and DINP, before and after a secondderivative treatment, respectively.

The NEUROSIM/L (version 3.2) software program (Fujitsu Ltd., Tokyo, Japan) was employed for training of a neural network. In comparison, we employed a principal-component analysis (PCA) to make a qualitative model which discriminates PVCs with different plasticizers, while a partial least-squares (PLS) regression was used to prepare a calibration model which predicts the plasticizer contents in PVC. The Pirouette (version 3.0) software program (Infometrix Inc., WA, USA) was applied for the PLS regression. In the neural network and PLS regression analysis, a leave-one-out cross-validation was performed.

\section{Architecture of neural networks}

The neural network was of a three-layered model. The input, intermediate (hidden), and output layers were composed of 120 , 5 , and 5 or 1 unit(s) (neuron(s)), respectively. Training the networks was carried out using an error back-propagation algorithm in this study. The maximum and minimum densities were normalized to 0.8 and -0.8 , respectively, as supervised signals. Training was ended when the maximum error converged to less than 0.1 .

\section{Results and Discussion}

PCA and neural-network analysis for the spectra of 41 kinds of PVC samples

Figure 2 illustrates a score plot of factor 1, factor 2 and factor 3 of PCA. The 123 spectra could be divided into four groups, namely DINP and DOP, TOTM, DOA and Polyester. Briefly, five plasticizers were divided into four groups (plasticizers) by PCA. As shown in Fig. 1, the NIR spectra of the PVC with DINP and that with DOP are similar to each other. And their second-derivative spectra are also similar to each other. Thus, it is not easy to discriminate them. With an increase in the contents of plasticizers, each point spreads in the top of PVC without a plasticizer. Figure 3 shows a score plot of PCA factor 1 and factor 2 for PVC with DINP and that with DOP. In the case of only PVC with DINP and that with DOP, 2 samples can be clearly discriminated. Factor 1 tends to decrease with an increase in the contents of plasticizers.

The case in where neural-network analysis was carried out, the 123 spectra could be divided into five groups by only one operation. Because the neural-network analysis is a typical nonlinear analytical method, little differences in the spectra for 2 plasticizers can be divided flexibly.

\section{Prediction of plasticizer content in PVC}

Figure 4 depicts the relationship between the actual values of the contents of DINP and their predicted values by neuralnetwork analysis from the second-derivative spectra. A good straight line could be obtained between the actual and predicted values.

Overfitting is considered when there are more unknowns than the number of data. However, the possibility of overfitting was low in this study. Generally, in the case of overfitting, the prediction rate is improved when the unknown is reduced. ${ }^{18}$ This was not improved, even if the input unit was reduced.

By way of a comparison, we made a calibration model by PLS regression. A PLS regression model with two factors for predicting contents of DINP in PVC from the second-derivative spectra is presented in Fig. 5. A good straight line could also be obtained. Table 2 summarizes the correlation coefficient (R) 

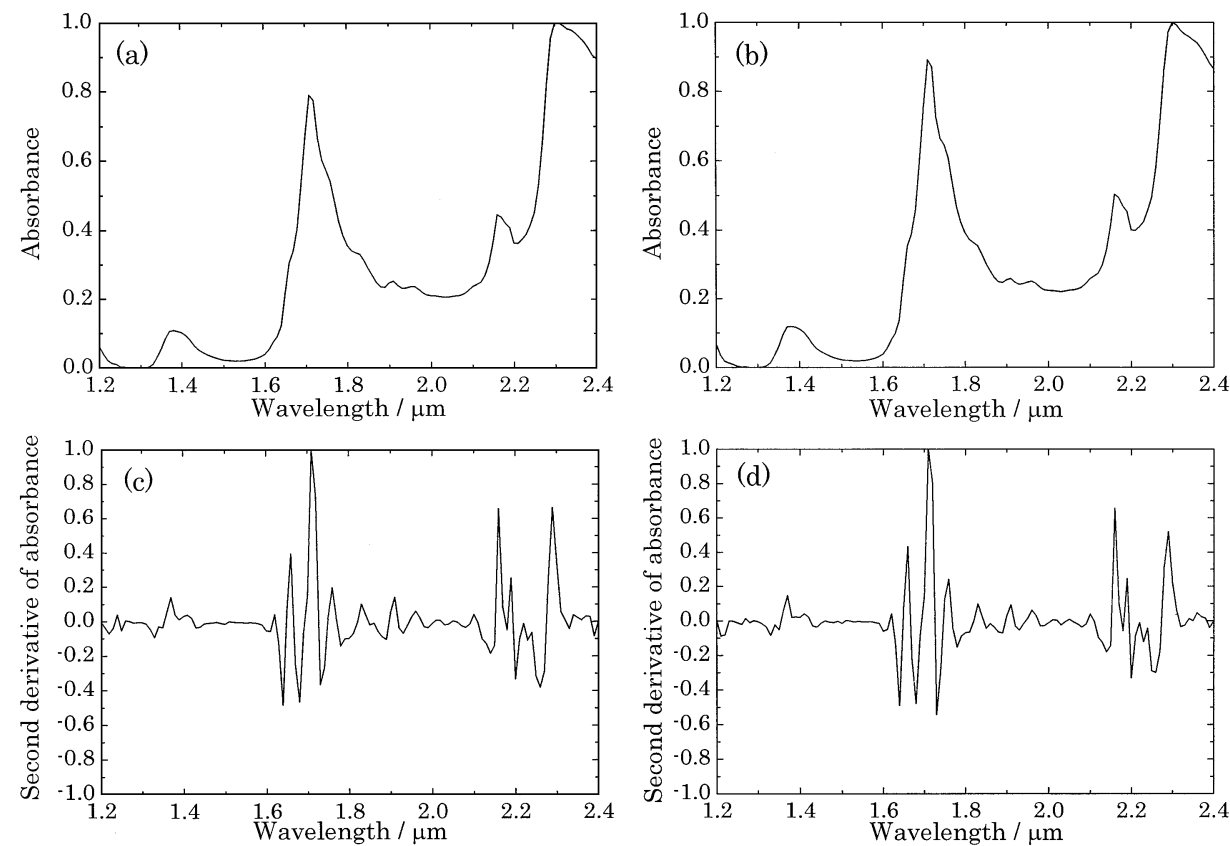

Fig. 1 Normalized spectra from PVC with a DINP content of $49 \mathrm{wt} \%$ (a), its second-derivative spectra (c), normalized spectra from PVC with a DOP content of $49 \mathrm{wt} \%$ (b) and its secondderivative spectra (d).

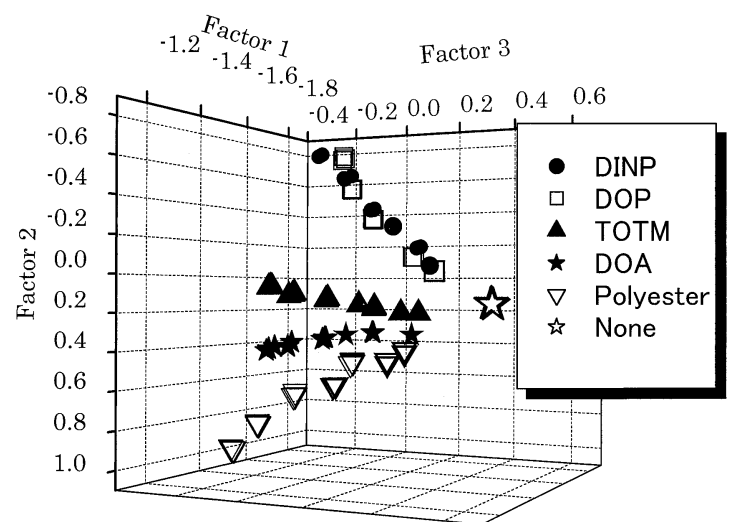

Fig. 2 Score plot of a principal-component analysis of factor 1 , factor 2 and factor 3 for DINP, DOP, TOTM, DOA or Polyester in PVC samples.

and the root-mean-square errors of prediction (RMSEP) for all plasticizers. Table shows that the neural-network analysis gives much better results in both the correlation coefficient and RMSEP than the PLS analysis.

The neural-network analysis is one of the most typical nonlinear analytical methods, while PLS is a linear method. The neural-network analysis flexibly deals with the change in the spectrum due to differences between the manufacturers and grades. For this reason, it is considered that the neural-network analysis gives much better results than the PLS analysis.

\section{Comparison with established methods}

Plasticizers in PVC are generally measured by GC, MS and NMR analyses. These methods need some sample preparation before analysis. Since a solvent is usually needed for those methods, it is necessary to dispose these solvents. NIR spectroscopy does not have stronger absorption. Overtone and

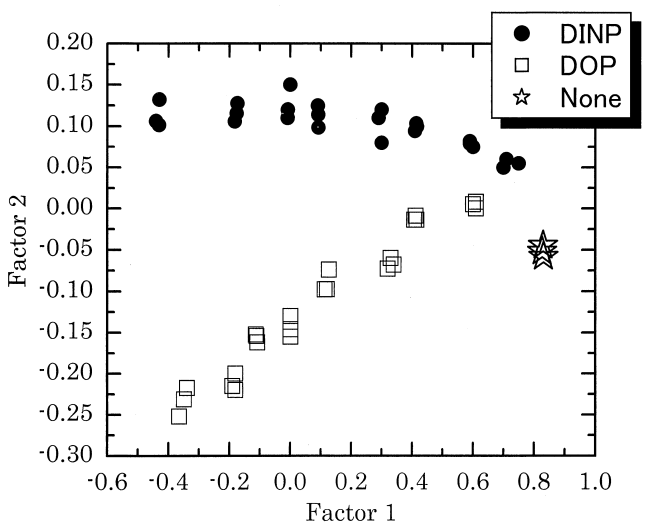

Fig. 3 Score plot of a principal-component analysis of factor 1 and factor 2 for DINP or DOP in PVC samples.

combination bands appear with adequate intensities in the NIR spectra. The NIR spectra can be measured quickly and nondestructively without sample preparation. Thus a solvent is not necessary in this method. Neural-network analysis is very useful for spectral identification. ${ }^{14}$ Neural networks automatically pick out and store features of the spectra. Slight differences in the spectra can be discriminated by a neuralnetwork analysis. The combined method can be carried out quickly and non-destructively. It is suitable for plastic recycling.

\section{Conclusions}

The present paper reports on the usefulness of NIR spectroscopy in combination with a neural-network analysis method in the qualitative and quantitative analysis of plasticizers in PVC. The 123 second-derivative spectra of 41 


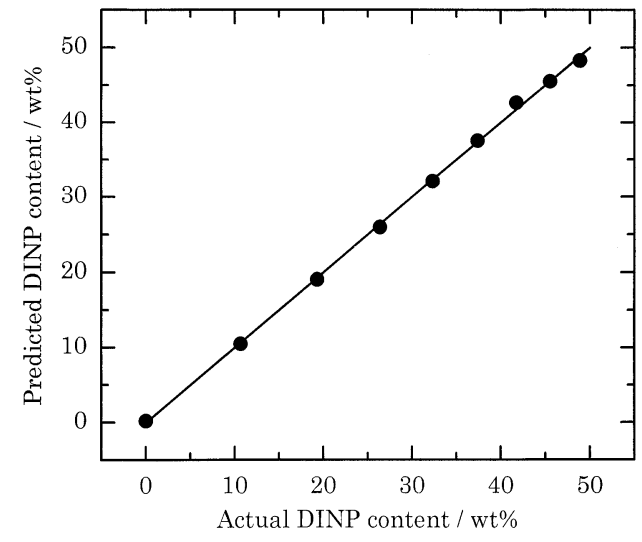

Fig. 4 Neural network analysis for predicting the DINP contents from the second-derivative spectra.

kinds of PVC samples could be discriminated steadily by neural-network analysis. A calibration model for predicting the plasticizer content in PVC was made using neural-network analysis. It was found that NIR and the neural network method could predict the plasticizer content. The correlation coefficients of DINP, DOP, TOTM, DOA and Polyester are $0.999,0.999,0.999,1.000$ and 0.999 , respectively. Their RMSEP are $0.41,0.41,0.52,0.42$ and 0.46 , respectively. A neural-network analysis gives better results than a PLS regression analysis.

The present result is quite important from the point of plastic recycling. Our technique can be applied rapidly and nondestructively. This combined technique using NIR spectroscopy and neural-network analysis is helpful in the material recycling of plastics. In this study, the possibility to develop a practical plastic discrimination system using the above approach was demonstrated.

\section{Acknowledgements}

The authors wish to thank Mr. Y. Nabeshima, Mr. Y. Miura and Mr. N. Muro of TOYOX CO., LTD. (Toyama, Japan) for providing the PVC samples.

\section{References}

1. H. W. Siesler and K. Holland-Moriz, "Infrared and Raman Spectroscopy of Polymers", 1980, Marcel Dekker Inc., New York.

2. L. G. Weyer, Appl. Spectrosc. Rev., 1985, $20,1$.

3. E. Stark, K. Luchter, and M. Margoshes, Appl. Spectrosc. Rev., 1986, 22, 335.

4. K. B. Whetsel, Appl. Spectrosc. Rev., 1986, $21,1$.

5. C. E. Miller, Appl. Spectrosc. Rev., 1991, 26, 277.

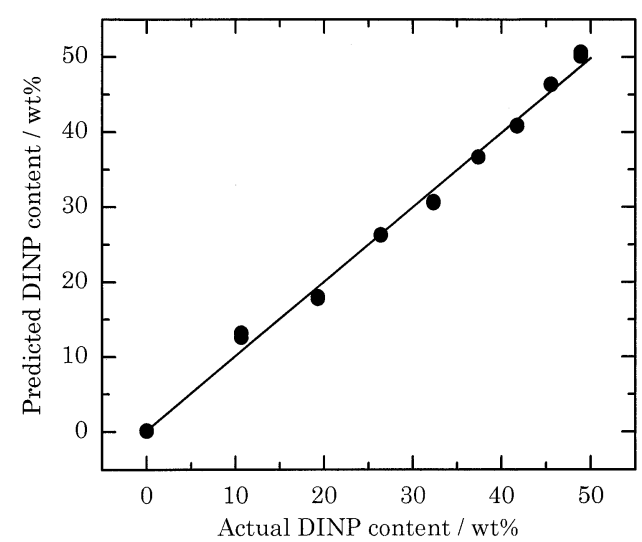

Fig. 5 Partial least-squares regression calibration model with 2 factors for predicting the DINP contents from the second-derivative spectra.

Table 2 Correlation coefficient (R) and root mean square error of prediction (RMSEP)

\begin{tabular}{lcccc}
\hline & \multicolumn{2}{c}{ Neural networks } & \multicolumn{2}{c}{ PLS regression } \\
& $\mathrm{R}$ & RMSEP & $\mathrm{R}$ & RMSEP \\
\hline DINP & 0.999 & 0.41 & 0.993 & 1.27 \\
DOP & 0.999 & 0.41 & 0.995 & 1.10 \\
DOA & 0.999 & 0.52 & 0.991 & 1.52 \\
TOTM & 1.000 & 0.42 & 0.993 & 1.27 \\
Polyester & 0.999 & 0.46 & 0.994 & 1.22 \\
\hline
\end{tabular}

6. H. W. Siesler, Macromol. Chem. Macromo. Symp., 1991, 5, 113.

7. "Handbook of Near-Infrared Analysis", ed. D. A. Burns and E. W. Ciurcziak, 1992, Marcel Dekker, New York.

8. J. W. Hall, D. E. Grzybowski, and S. L. Monfre, J. NIR Spectrosc., 1993, 1, 55.

9. K. A. B. Lee, Appl. Spectrosc. Rev., 1993, 28, 231.

10. K. Molt and D. Ihlbrock, Fresenius J. Anal. Chem., 1994, $348,523$.

11. G. Lachenal, Vib. Spectrosc., 1995, 9, 93.

12. M. P. B. van Uum, H. Lammers, and J. P. de Kleijin, Macromol. Chem. Phys., 1995, 196, 2023.

13. A. Khettry and M. G. Hansen, Polym. Eng. Sci., 1996, 36, 1232.

14. J. Zupan and J. Gasteiger, "Neural Networks for Chemists", 1993, VCH, Weinheim.

15. B. K. Lavine, Anal. Chem., 1998, 70, 209R.

16. T. Matsumoto, K. Tanabe, K. Saeki, T. Amano, and H. Uesaka, Bunseki Kagaku, 1999, 48, 483.

17. K. Saeki, T. Matsumoto, K. Tanabe, and T. Amano, Bunseki Kagaku, 2001, 50, 223.

18. K. Tanabe and T. Matsumoto, J. Comput. Chem. Jpn., 2002, 1, 23. 